\title{
Security for the core network of third generation mobile systems
}

\author{
GÜNTER HORN, DIRK KRÖSELBERG \\ Siemens AG, Corporate Technology, D-81730 Muenchen, Germany
}

\author{
STEFAN PÜTZ \\ T-Mobil, P.O. Box 300463, D-53184 Bonn, Germany
}

\author{
ROLAND SCHMITZ \\ T-Nova Technology Centre, D-64307 Darmstadt, Germany
}

Keywords: $\quad$ UMTS, MAP Security, Multimedia domain, SIP, IPSec, IKE, Key Management

Abstract: This contribution gives a survey of the present standardisation activities by 3GPP ( $3^{\text {rd }}$ Generation Partnership Project ${ }^{1}$ ) in the area of security for signalling in the core network of third generation mobile systems. We give an overview of the protocols that need to be secured, present the basic principles behind the overall security architecture and describe the key management and format of secured messages, as far as they have already been finalised. In particular, we address core network security aspects of the 3GPP multimedia domain.

13 GPP was formed by regional standards organisations from Europe, Asia and North America to produce specifications for a third generation mobile system named UMTS which is designed to evolve from GSM core network. There is a competing effort known as 3GPP2 with partners from North America and Asia. 


\section{THREATS TO CORE NETWORK SECURITY FOR MOBILE RADIO NETWORKS}

The core network of mobile radio systems is the part of the network which is independent of the radio interface technology of the mobile terminal. It is used for transporting user data as well as signalling commands needed to ensure smooth operation of the overall system. Although eavesdropping or modifying signalling messages in the core network would have serious consequences for mobile radio systems because sensitive authentication data of mobile subscribers are transported across the core network, in second generation (2G) mobile systems like GSM the core network is not secured. If an intruder succeeded in eavesdropping on these authentication data, serious impersonation attacks or eavesdropping on user traffic on the air interface might result. To date, however, no such attacks on GSM core networks are known.

One reason for not securing the core network of $2 \mathrm{G}$ mobile systems was the initial design goal of making $2 \mathrm{G}$ mobile systems only as secure as fixed network connections which meant in effect that security features of second generation systems were basically constrained to the most vulnerable part of the network, the radio interface. A second, equally important, reason was that the core network was considered "closed", i.e. without external interfaces which might be used by attackers. GSM uses the SS7 protocol stack with MAP (Mobile Application Part) as an application on top for its signalling messages. Due to its complexity and the limited availability of implementations, detailed knowledge of SS7 is confined to telecoms insiders which helps to reduce the number of potential attackers.

All these assumptions, however, do no longer hold for third generation systems such as UMTS: with the introduction of IP-based transport to most, if not all, interfaces of the UMTS network reference model new vulnerabilities of the core network as well as new potential threats directed towards the core network from the outside have to be taken into account. In addition to building, and managing, their own "private" transport networks, operators also have the technical possibility to rent the transport capacity required between any two nodes of the reference model from virtually any ISP offering real-time transport services.

For these reasons, the security features of UMTS will also cover the core network. Protection is provided for only signalling messages, as attacks on these seem to pose the greatest risk. Protection of user data is only provided by the UMTS standard in the access network. If protection of user data is also required in the core network it is to be covered by end-to-end security at 
an application layer above the $3 \mathrm{G}$ core network which is considered outside the scope of the UMTS standard.

The present paper describes the security architecture, key management techniques and message formats for the core network signalling security of the $3^{\text {rd }}$ generation mobile radio system UMTS as currently specified by the security group of 3GPP. An earlier paper [Pütz] already described key management schemes and message formats for providing security for MAP (Mobile Application Part, see section 2.1) as discussed in 3GPP during 1999. Our present contribution shows how considerations have evolved since then. Here, we also cover core network security aspects of the new IP-based multimedia domain of UMTS and focus on the different approaches for a core network key management architecture presented within the 3GPP security group to provide key management for SS7-based as well as IP-based security protocols. The overall goal of the architecture is to secure both SS7based and IP-based signalling messages in the core network.

\section{OVERVIEW OF THE CORE NETWORK : SS7- AND IP-BASED PROTOCOLS}

In what follows, we give a very brief overview over the signalling protocols used in the current $2 \mathrm{G}$ core networks. For more detailed information on the protocols mentioned here, the reader should consult the book [Walke]. These protocols will continue to be used in $3 \mathrm{G}$ core networks, with the difference that in $3 \mathrm{G}$ networks security features for these protocols will be available.

\subsection{SS7-based Protocols: MAP and CAP}

The signalling between the various network elements of a mobile core network (e.g. Base Station Subsystem (BSS), Mobile Switching Center (MSC), Home Location Register (HLR), Visitor Location Register (VLR) and Authentication Center (AuC)) of the GSM system are based on the Common Channel Signalling System No. 7 (SS7). A mobile-specific application protocol, the Mobile Application Part (MAP), was developed on top of the SS7 protocol stack to accommodate the mobile-specific signalling in a GSM network. The MAP protocol is implemented in all switching centers directly linked to the mobile network. In GSM, for example, the user authentication data (Challenge RAND, Signed Response SRES and Session Key $\mathrm{Kc}$ ) are transported by MAP dialogues from the HLR to the VLR. 
In addition, a protocol called CAMEL (Customized Applications for Mobile Network Enhanced Logic) [GSM 03.78] is used in GSM to accommodate the wish of mobile operators to offer operator-specific services to their customers. CAP, the CAMEL Application Part, is currently also being transferred between network elements as an application on top of the SS7 protocol stack.

3GPP has based its specifications for UMTS, e.g. [3G TR 29.002], on an evolved GSM core network, thereby retaining the basic core network architecture of GSM and its protocols. It is envisaged that the SS7-based transport stack for MAP and CAP will be gradually replaced with an IPbased transport stack in the UMTS core network. But there will be quite a long period where nodes supporting MAP or CAP over IP will have to communicate with nodes supporting MAP or CAP over SS7, e.g. nodes in a $2 \mathrm{G}$ network. In what follows, we will refer to these application protocols that may be run either over SS7 or over IP as "legacy protocols".

\subsection{IP-based Protocols: GTP}

Recently, a packet-oriented service concept for the transfer of data in $2 \mathrm{G}$ networks has been introduced: General Packet Radio Service (GPRS) [3G TS 29.060]. Implementing the packet-oriented technique of GPRS in the core network of GSM already constitutes a major step towards UMTS where the packet service (PS) domain is realized using GPRS. The most important new network elements needed for the packet-oriented GPRS core network are the Serving GPRS Support Node (SGSN) providing the mobile station with functional support in the core network and the Gateway GPRS Support Node (GGSN). The GGSN is located at the border of the IP-based GPRS core network and serves as the interface to external networks. SGSNs and GGSNs communicate by using the GPRS Tunneling Protocol (GTP) which encapsulates data packets and all relevant signalling information. The GPRS backbone protocol is IP, with GTP running on top of it. We therefore refer to GTP as a "native IP protocol" in the sequel.

\section{OVERVIEW OF THE UMTS IP-BASED MULTIMEDIA DOMAIN (IM DOMAIN)}

The UMTS IM domain, currently being specified by 3GPP and not yet due for the first release of UMTS, will offer voice over IP and multimedia services which are based on the concept of a "call". Only IP-based protocols are used in the IM domain. For transport of both signalling and user data 
within UMTS the packet services of the packet switched domain are used. In order to realize calls, an IP-based call signalling protocol is needed. 3GPP selected SIP (Session Initiation Protocol, cf. [RFC 2543]) for this purpose. SIP is a client-server protocol supporting proxy functionality. Like HTTP, it uses a human-readable text representation for its messages. The mobile terminal contains a SIP user agent (UA) which communicates with so-called Call State Control Functions (CSCFs) at the network side. These network entities offer SIP proxy/server functionality. In order to support mobility, several types of CSCFs, residing either in the visited or in the home network, and a Home Subscriber System (HSS) have been defined. For details the reader is referred to [3G TS 23.228].

\section{SECURITY FOR SS7 AND MIXED SS7/IP-BASED PROTOCOLS}

\subsection{The basic principles}

This section is discussing only MAP as the risk associated with an attack on the MAP protocol seems to be much higher than the related risk for the CAP protocol. But the means to secure CAP would be identical.

In the future, MAP may use SS7-based or IP-based transport. In order to secure MAP over IP, there are basically two options:

- Provide security at the MAP application layer

- Provide security at the network layer by using IPSec

For MAP over SS7 it is only possible to provide security at the application layer. A different solution would mean to introduce security into the SS7 stack, a formidable task which would affect many other applications also using SS7, but not requiring security. Hence, if security was provided at the network layer for MAP over IP, a need for application-to-network layer security gateways (ANLSG) would arise when interworking between IP and SS7 transport became necessary. Such an application-to-network layer gateway would have to translate application layer MAP security (in the SS7 domain) into network layer security (in the IP domain). This is highly undesirable for several reasons:

- There is high additional complexity introduced by such a gateway.

- To receive protected MAP messages and to transform them into IPsec secured messages, an ANLSG /IP gateway must be capable of terminating application layer (MAP) security on the SS7 side. Since 
MAP routing is based on the IMSI number and does not happen at the MAP-layer, an SS7 end-entity cannot directly address (and usually does not even know) gateways at the network layer or other MAP entities. Therefore, it seems to be difficult to set up a MAP security association between a MAP end-entity and an ANLSG.

- The trust issues raised by this solution are difficult. The endpoints of the MAP communication would have to trust the ANLSG. But a MAP over SS7 node cannot even know which gateway the MAP messages pass. ANLSGs could even be located in intermediate networks, e.g. if the originating network has no direct link to the IP world. So ANLSGs were likely to influence and even restrict the world-wide PLMN topology, in order to guarantee a closed chain of trust between all communicating MAP entities.

This speaks in favour of providing security at the application layer also for MAP over IP. Protocols that can be carried by both SS7 and IP should therefore be secured at the application layer. A further advantage of this approach is that no additional specification and implementation effort is foreseen for MAP security when IP-based transport for MAP is introduced.

\subsection{Key Management}

The proposed mechanism consists of a two-tiered key management architecture. The overall picture is similar to that presented in [Pütz]: a new network element, the Key Administration Center (KAC), is introduced in every network. KACs communicate with each other over the $Z_{A}$ interface (see Figure 1). In particular, KACs negotiate MAP SAs (Security Associations), which are network-wide SAs, i.e. the SAs are valid for a specific pair of networks for a certain period. Network-wide SAs are necessary because, in general, it is not possible for a sending MAP network entity in a network $A$ to determine the address of the receiving network entity in a network B. This is due to the particularities of routing for MAP over SS7 which is done by intermediate gateways based on the user identity IMSI. The address of the receiving network entity may change without the world outside network B knowing, e.g due to a user's records being moved from one machine to another one within network $B$. Then the user's identity would point to that other machine. MAP SAs include the following information:

- Encryption and MAC Algorithm Identifier

Identifies the encryption and MAC algorithms and their mode of operation used for confidentiality and integrity protection. It was agreed to mandate the use of the AES for both purposes. The modes remain to be specified. 
- Encryption Key and MAC Key

Keys to be used for confidentiality and integrity protection of messages exchanged between networks A and B.

- MAP Protection Profile reference

This field gives a reference to the chosen MAP protection profile. A MAP Protection Profile (MAP PP), is an specification of how MAP operations over the $Z_{C}$ interface shall be protected. It indicates whether a MAP operation needs protection, and if so, indicates the protection mode (cf. section 4.3) to be used.

- Fallback to Unprotected Mode Indicator In case protection is required, this parameter indicates whether fallback to unprotected mode is allowed.

- SA Lifetime

In contrast to IPSec SAs, the lifetime of a MAP SA has to be given in absolute time because the MAP SA is network-wide and not all MAP entities in a network necessarily receive the MAP SA from the KAC at the same time.

The MAP SAs specify how to protect the communication over the $Z_{C}$ interfaces between two network elements (NE). KACs also distribute the SAs to the relevant NEs via the $Z_{B}$ interface. NEs then use the distributed SAs for the actual secure MAP signalling message transfer over the $Z_{C}$ interface.

The overall scheme is shown in the following picture:

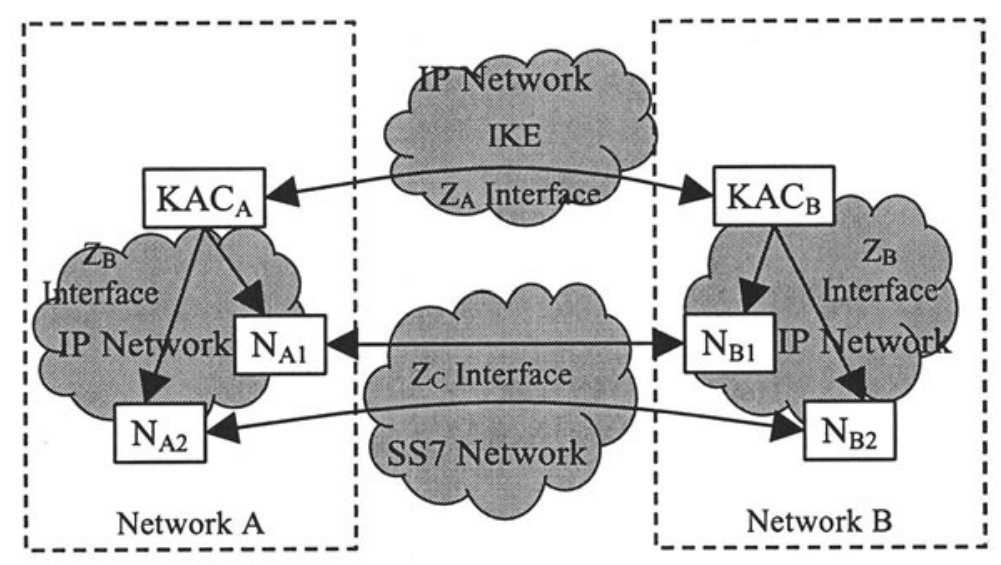

Figure 1: Overall Key Management Scheme for Secure Legacy Protocols 
For the secure negotiation of MAP SAs over the $Z_{\mathrm{A}}$ Interface, IKE, the Internet Key Exchange Protocol [RFC 2409] will be used together with a newly defined Domain of Interpretation (DoI) for MAP which has recently been submitted as a draft to the IETF [Arkko]. In the initial phase of the deployment of MAP Security in $3 \mathrm{G}$ core networks, authentication in IKE over the $\mathrm{Z}_{\mathrm{A}}$ Interface will be based on pre-shared secrets that can be exchanged between network operators in the course of roaming agreements. For a later phase, certificate-based mechanisms are envisaged (cf. section 5).

For the secure distribution of MAP SAs from the KACs to the NEs over the $Z_{B}$ interface, also an IPSec-based mechanism is envisaged, but not yet fully specified. In particular, it is still to be decided whether the distribution mechanism will be based on a "pull" or a "push" approach, i.e. if the network elements have to request the MAP SAs from their respective KACs before they can start communicating or if the MAP SAs are distributed by the KACs beforehand. The format of secured MAP messages exchanged over the $\mathrm{Z}_{\mathrm{C}}$-interface between NEs is discussed in the next subchapter.

\subsection{Message Formats for Secure MAP}

This subchapter describes mechanisms for establishing secure signalling links between network nodes which communicate over the $Z_{C}$ Interface using the MAP protocol.

\subsubsection{General Structure of Secured MAP Messages}

Secured MAP operations are performed via the MAP protocol in the course of secured MAP dialogues. For secured MAP operations, three levels of protection (or protection modes) are defined providing the following security features (cf. also [Pütz]):

- Protection Mode 0: no protection

- Protection Mode 1: integrity

- Protection Mode 2: integrity and confidentiality

Secured MAP messages consist of a MAP message header, a security header and the protected payload that is the result of applying the corresponding protection mode to the original MAP message payload.

In all three protection modes, the security header is transmitted in cleartext. Among other pieces of information, it contains the sending network identity and the Security Parameter Index (SPI), an arbitrary 32-bit value that is used in combination with the sending network identity to uniquely identify a MAP SA. 


\subsubsection{Format of Secured MAP Message Body}

Since protection mode 0 offers no protection at all, the protected payload in protection mode 0 is identical to the original MAP operation payload in cleartext.

Of the two remaining protection modes, only protection mode 2 is presented here in detail as an example. For more details we refer the reader to [3G TR 33.800]. The secured MAP message body in protection mode 2 takes the following form:

\section{TVP $\| \mathrm{E}_{\mathrm{KSXY}(\text { con) }}\left(\right.$ cleartext) $\| \mathrm{H}_{\mathrm{KSXY} \text { (int) }}(\mathrm{TVP} \|$ MAP header $\|$ security header $\|$ $\mathrm{E}_{\mathrm{KSXY} \text { (con) }}$ (cleartext))}

where "cleartext" is the original MAP message in clear text. Message confidentiality is achieved by encrypting cleartext with the confidentiality session key $\mathrm{KS}_{\mathrm{XY}(\mathrm{con})}$. Authentication of origin and message integrity are achieved by applying the message authentication code (MAC) function $\mathrm{H}$ with the integrity session key $\mathrm{KS}_{\mathrm{XY} \text { (int) }}$ to the concatenation of Time Variant Parameter TVP, MAP header, security header and the encrypted clear text $\mathrm{E}_{\mathrm{KSXY} \text { (con) }}$ (cleartext).

The TVP used for replay protection of secured MAP messages is a 32 bit time-stamp. The receiving network entity will accept a message only if the time-stamp is within a certain time-window. The resolution of the clock from which the time-stamp is derived must be agreed as a system parameter, the size of the time-window at the receiving network entity does not need to be standardised.

\section{SECURITY FOR NATIVE IP-BASED SIGNALLING PROTOCOLS}

There are many possible, well-known threats for an IP-based core network running native IP protocols. An IP-based UMTS core network does not make sense without security measures. This chapter gives an overview of the IP core network security architecture currently standardized by 3 GPP.

\subsection{Basic Architecture}

Security for IP-based signalling protocols can be implemented at different layers. The application protocols themselves often include 
mechanisms to apply integrity or confidentiality to their messages. But these application layer security mechanisms in many cases only provide a basic level of protection, e.g. password-based schemes, or they are not flexible enough in some situations. But even more importantly, applying protection at the application layer would probably require the support of many different security mechanisms in the UMTS core network.

At the transport layer the TLS protocol [RFC 2246] is a security protocol with wide acceptance in the internet community for securing application protocols like HTTP. However, TLS can only be used together with TCP and therefore is not suited for a security architecture encompassing the whole UMTS core network. (The SIP protocol, e.g. requires the support of UDP.)

Based on these considerations, the 3GPP security group decided to apply core network signalling security at the network layer. For securing the IP protocol, the IETF specified the already well-established IPsec framework [RFC 2401] which offers connection-less integrity protection and confidentiality combined with a mechanism against replay attacks through the base protocols AH [RFC 2402] and ESP [RFC 2406]. Automated key management is provided by the Internet Key Exchange protocol IKE [RFC 2409].

The decision to secure the IP-based core network signalling with IPsec allows for a clear security architecture which is outlined in the following:

All IP communication that requires to be protected will be routed in a hop-by-hop fashion through protected tunnels. To support this approach between different networks, a new logical entity called security gateway (SEG) has been added to the architecture. 


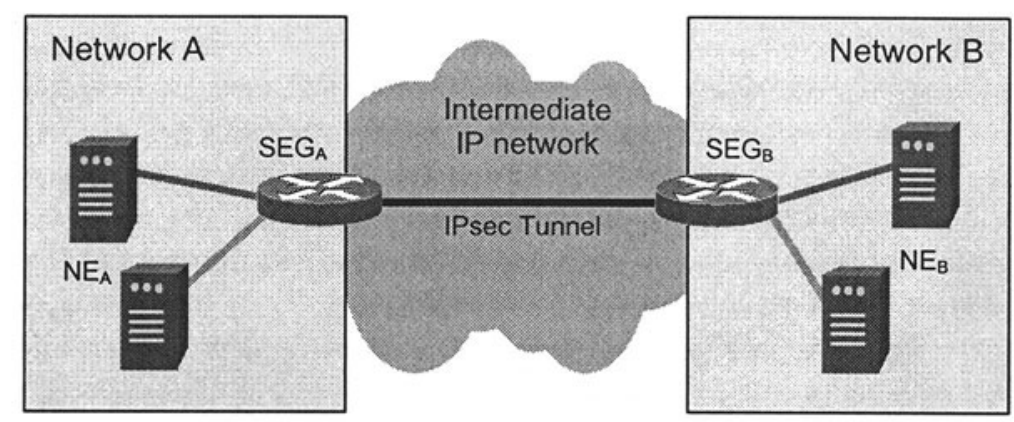

Figure 2: Secure IP inter-network communication via SEG entities

SEGs operate at the border of a network, providing IP security for internetwork IP traffic. SEGs establish and maintain IPsec tunnels with any network entity (NE) of their own network that uses this SEG to secure intranetwork IP traffic destined for other networks. Similarly, IPSec tunnels may be established between two NEs residing in the same network. Depending on the network configuration the SEG entities support a single, uniform IPsec tunnel to another network that tunnels all types of IP communication, or they establish several tunnels applying different security services for different protocols, ports or even hosts. Policy information for these secure tunnels has to be exchanged in advance, as part of the roaming agreement, between the operator's SEG entities.

The first UMTS core network protocol that will be secured by IPsec is the IP-based GTP protocol, cf. section 2.2. GTP consists of a control part, GTP-C, that transports signalling messages between different GPRS entities, and a user part, GTP-U, that transports the tunnelled IP user data. Between two adjacent GPRS entities, all GTP-U traffic uses the same ports, and consequently all or no GTP-U traffic between two GPRS entities could be secured using IPSec. In line with the general approach to secure only core network signalling, only GTP-C will be secured. The SEG entities will provide the functionality of GPRS border gateways which were introduced in the GPRS specification as the entities to enforce secure GTP traffic at the network border.

\subsection{Core network security aspects for the IM domain}

From a security point of view, it is noteworthy that the usual distinction between user and signalling traffic within the mobile network is not given 
any more. SIP signalling messages are transported as IP packets from and to the mobile user as part of the user payload within the GPRS bearer, not over a separate signalling channel. In contrast to signalling, the GPRS user plane will not be secured. Due to these considerations, additional protection has to be provided to SIP signalling messages carried in the UMTS packet domain. Security for the IM domain is divided into two different domains:

- IM access domain, which extends between the mobile terminal and an appropriate CSCF that is responsible for protecting the access to network resources.

- IM network domain which extends from this CSCF further into the network.

Protection for signalling in the IM access domain may be provided either at the application layer or at the network layer by applying IPSec to IP packets sent between mobile terminal and CSCF, encapsulated en route by GTP where required.

On the interfaces which belong to the IM network domain GTP is not used, and IM domain signalling messages can be distinguished at the network layer by their port number, hence they can be selectively protected by IPSec. In particular, the transfer of authentication data from the HSS to the appropriate CSCF can be protected in this way.

Using this approach allows for a complete protection of IM domain signalling between the mobile terminal and the network as well as on all core network interfaces over which the messages are sent. Another advantage here is that the security is not affected by or depending on the application protocol between CSCF and HSS.

Work on the security for the IM domain is still ongoing within 3GPP, with many aspects not yet decided.

\subsection{IPsec configuration}

The IPsec framework defines a flexible but complex security architecture. Although flexibility is important, each integration of IPsec requires decisions for modes of operation, security algorithms and combination of protocol features that affect the overall system security. Since complexity stands against security, the 3GPP security group has agreed to recommend the following restrictions or simplifications for the application of IPsec:

- To simplify the IPsec options, it is recommended to use the ESP protocol in tunnel mode. The AH protocol and transport mode can be used, but implementations are not required to support these.

- IP payload compression is regarded as inefficient when applied within the 3GPP core network and its usage is therefore not recommended. 
- According to the IPsec standards, for ESP it is legal to use encryption only, without integrity protection. Since the ESP header is unprotected without integrity protection, and it contains a sequence number used for IPsec replay protection, replay protection cannot operate securely and therefore cannot be used. Hence, it is mandated to never use ESP without integrity protection.

- Since the DES cipher algorithm nowadays is not regarded secure any more, and DES is the only mandatory encryption algorithm in the IPsec specification, it is recommended to make AES mandatory for 3GPP instead. AES will soon become part of the IPsec specifications [Frankel].

\subsection{Key Management}

The key management architecture for MAP security introduces a new network entity (NE) called key administration center (KAC) as described in section 4. Each network includes a KAC to remotely negotiate MAP security associations on behalf of the MAP NEs. Within the 3GPP security group, the initial idea was to design a unified key management where the KACs also provide security associations for IPsec to support the IP-based NEs. This approach proved to be difficult. Concerns about its feasibility stem from the fact that on the one hand addressing for IP communication is quite different from MAP addressing. This implies that, for IPSec, individual security associations need to be established peer-to-peer between two NEs. On the other hand, SA negotiation in the IPsec standards is intended not to take place remotely by a third party, but directly between the IPsec peers that use these SAs subsequently. When using Key Administration Centers, renewal of SAs and SA delete notifications must be supported as well by the KACs. While these difficulties may have been possible to solve the general feeling was that a certificate-based flat key management architecture as described below was the way to go in the future anyhow. It was felt that an expensive KAC-based solution with only a limited lifetime was difficult to justify. As a consequence, 3GPP decided not to use the KACs for IPsec SA negotiation, but to support direct SA negotiation between IPsec hosts.

Between different networks, it is mandated that all IP traffic passes an SEG at each network border. SAs between two networks are therefore only required between specific SEGs that connect the networks. These SEGs support the internet key exchange protocol IKE, which is the default key management protocol for IPsec. With IKE, the SEGs can negotiate their IPsec SAs directly. Standard IPsec implementations can be used here.

The IKE protocol allows to dynamically negotiate security association including session keys, but nevertheless requires the peers to authenticate 
themselves during the initial key exchange. This authentication can be based on a symmetric key between the peers (shared secret), or on public key techniques with or without certificates. In a first phase, two operators can rely on the off-line exchange of a pre-shared key between two SEGs as part of the roaming agreement that is subsequently used for authenticating their SEGs within IKE. Similarly, manual key management could be assumed between an SEG and the NEs or between two NEs in the same network. This static keying method is quite feasible as long as there are only few SEG-SEG connections between two network operators and a quite limited number of NEs in a network. (The latter is the case at least in the initial phase of a UMTS network.)

With growing network complexity, this approach to key management seems no longer feasible, and a certificate-based approach becomes necessary. In the following paragraph we sketch a PKI-based architecture for the UMTS core network which can provide automated inter-network key management for IPsec without KAC functionality and is therefore called flat key management. Work on such an architecture to be used in the UMTS core network has just started.

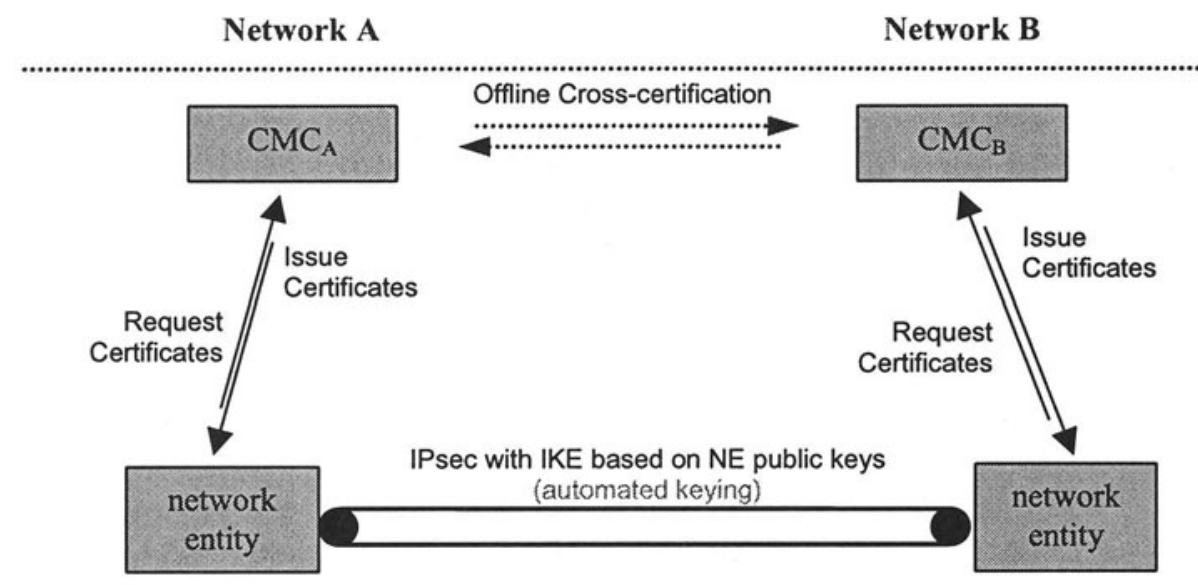

Figure 3: Flat key management for the UMTS core network

A central entity in a flat key management architecture is a certification management center (CMC) which at least includes a certification authority (CA) to issue public/private key pairs and certificates for network entities like SEGs and a repository serving as certificate database. It should also provide a mechanism to handle the revocation of certificates. There are several possibilities, cf. [Preneel]. A well-known example is OCSP (online certificate status protocol), [RFC 2560] defined by the IETF PKIX 
workgroup, but other alternatives for revocation mechanisms need to be considered as well. Of course, not all functions of a CMC need to reside in the same physical entity.

Trust relations between different networks with a roaming agreement are required within this model. These are established by a cross-certification between the networks, where the CMC of network I issues a certificate for the CMC of network II and vice versa. IKE, running directly between the SEGs can use such certificates for authentication and key agreement. Instead of cross-certifying each single pair of networks with a roaming agreement, a world-wide top-level certification authority (CA) run by an association of UMTS operators could be established, that certifies the public keys of the CAs in each network. Provided that all network operators have trust in the top-level CA, any network can accept the certificate of any other network as trusted within this hierarchical model. Although theoretically sound and scaleable, setting up a PKI is widely regarded as being a difficult task. Since there is no need for supporting a complex inter-network IP architecture for the first release of $3 \mathrm{G}$ networks, it seems inappropriate to start with a fullblown architecture for a flat key management for core network security. Rather, this full-blown architecture is regarded as the solution for the medium and long term.

\section{CONCLUSIONS AND OUTLOOK}

The transition from the closed world of (unprotected) core networks in second generation mobile systems to the much more open IP-based core networks in third generation mobile systems increases the risk of attacks on these core networks considerably. Especially attacks on signalling messages have a high potential of damage. Therefore, core network signalling will be protected by appropriate security mechanisms in UMTS. Different mechanisms have to be provided for legacy protocols and native IP-based protocols.

UMTS specifications for core network signalling security are expected to be completed by the end of 2001, with the exception of work on a flat key management architecture for native IP-based protocols which has only started and is expected to extend well into 2002. 


\section{ACKNOWLEDGEMENT}

This contribution gave a survey of some of the present security standardisation activities by $3 \mathrm{GPP}$. The authors would like to thank all their colleagues in 3GPP who contributed to the specification of the results presented in this paper.

\section{REFERENCES}

[3G TR 29.002] 3GPP Technical Report ${ }^{2}$ 3G TR 29.002: Mobile Application Part (MAP) specification.

[3G TR 29.060] 3GPP Technical Report 3G TR 29.060: GPRS Tunnelling Protocol (GTP) across the $\mathrm{Gn}$ and $\mathrm{Gp}$ Interface.

[3G TR 33.800] 3GPP Technical Report 3G TR 33.800: Principles for Network Domain Security.

[3G TS 23.228] 3GPP Technical Specification 3G TS 23.228: Service requirements for the IP Multimedia Core Network Subsystem.

[Arkko] J. Arkko: The MAP Security Domain of Interpretation for ISAKMP, Internet Draft.

[Frankel] S. Frankel, R. Kelly, R. Glenn: The AES Cipher Algorithm and Its Use With Ipsec, Internet Draft.

[GSM 03.78] ETSI Technical Specification GSM 03.78: Customized Applications for Mobile Network Enhanced Logic (CAMEL).

[Preneel] B. Preneel et al.: A Taxonomy of Certificate Status Information Mechanisms, Proc. Information Security Solutions Europe (ISSE) 2000.

[RFC 2246] IETF RFC-22463: The TLS Protocol Version 1.0.

[RFC 2401] IETF RFC-2401: Security Architecture for the Internet Protocol.

[RFC 2402] IETF RFC-2402: IP Authentication Header (AH).

[RFC 2406] IETF RFC-2406: IP Encapsulating Security Payload (ESP).

[RFC 2409] IETF RFC-2409: The Internet Key Exchange (IKE).

[RFC 2543] IETF RFC-2543: SIP: Session Initiation Protocol.

[RFC 2560] IETF RFC-2560: X.509 Internet Public Key Infrastructure Online Certificate Status Protocol (OCSP).

[Pütz] S. Pütz, R. Schmitz, B. Tietz: Secure Transport of Authentication Data in Third Generation Mobile Phone Networks, Proc. of Secure Networking, CQRE (Secure) '99.

[Walke] B. Walke: Mobile Radio Networks, Wiley \& Sons, 1999.

2 GPP specifications and reports are available under www.3gpp.org.

${ }^{3}$ IETF RFCs and drafts are available under www.ietf.org. 